\title{
La relación médico-paciente en el siglo 21
}

\author{
The physician-patient relationship in the $21^{\text {st }}$ century
}

La relación médico-paciente es un tema clásico en la medicina y en la enseñanza médica. La imagen es la de una relación entre dos: un médico y un paciente, casi como en una isla desierta, en la cual participan una persona necesitada de ayuda por una dolencia o enfermedad y un profesional con conocimientos, capacidad y autoridad para curar o al menos para aliviar su enfermedad. El tipo tradicional de relación es un modelo paternalista, vertical, en el cual el médico actúa como un padre que cuida y protege al hijo necesitado, basado en una relación de confianza y de dependencia. Es evidente que este modelo es hoy inexistente. Aun cuando se pueda mantener cierta asimetría y dependencia, la relación paternalista que caracterizó a la medicina por al menos 25 siglos ha cambiado o casi ha desaparecido en los últimos 50 o 60 años. Cambió porque cambió todo: la sociedad, la ciencia, la medicina, el paciente y la forma en que éstas se relacionan. En este editorial nos proponemos analizar este cambio, y en especial el cambio que se vive o que se proyecta en la relación entre el paciente y el médico o la medicina en nuestro actual siglo 21.

Cabe recordar que ya en los siglos 18 y 19 surge en la medicina la preocupación por la persona del enfermo, se incorporan las dimensiones social y psicológica de los enfermos, comienzan a desarrollarse las especialidades médicas, surgen nuevas profesiones de la salud y la medicina evoluciona hacia la búsqueda de mayores fundamentos científicos. La sola experiencia del médico o maestro se considera insuficiente y se espera que las decisiones y procedimientos médicos tengan una base experimental. La medicina se hace ciencia y a la vez la ciencia se percibe en una esfera diferente a la de los valores. Consecuentemente el modelo paternalista de relación evoluciona hacia los modelos informativo, interpretativo y deliberativo ${ }^{1}$, en los cuales el paciente es quien toma sus decisiones, o al menos participa activamente en los procesos de decisión. Este cambio, sumado al hecho de la variedad y cantidad de especialidades médicas y de profesiones de la salud, ha llevado a que, actualmente, lo procedente es hablar de relación clínica y no de relación médico-paciente, pues en esta relación participan muchos agentes diferentes, cada uno desde su perspectiva y desde sus competencias ${ }^{2}$.

Pero los cambios que vivimos en las últimas décadas y que siguen ocurriendo a gran velocidad generan confusión en todos los ámbitos del pensamiento, análisis y predicciones. Los cambios y progresos en ciencia, tecnologías, comunicaciones, transporte, desplazamiento poblacional, producción industrial, etc., llevan a la conclusión de que en pocas décadas nos ha cambiado sustancialmente la vida. Efectivamente el cambio cultural es en todas las sociedades de tal magnitud que se ve reflejado en las motivaciones, valores, relaciones, tipos de familia, y en las decisiones personales ante una enfermedad. Las diferentes generaciones, desde los "tradicionalistas" nacidos entre 1900 y 1945 hasta los "millennials" nacidos entre los años 1981 y 2000, han llegado a su adultez con características $y$ criterios radicalmente diferentes. Este extraordinario cambio afecta indudablemente a la relación clínica y el desafio para los profesionales de la salud es aprender a responder a las necesidades de los pacientes, tanto de los tradicionales como a las de los que consideramos pacientes del futuro o del siglo 21, aun cuando ya hemos recorrido casi 1/5 de este siglo.

El paciente actual es una persona consciente de sus derechos, los que están legalmente protegidos en la mayoría de los países ${ }^{4}$, generalmente desconfiado, tremendamente exigente, con acceso fácil a información médica por internet, preocupado por los costos y de sus coberturas previsionales, con altas expectativas de soluciones rápidas para su enfermedad a través de una atención de excelencia con tecnología de última generación. Sin embargo, el paciente actual y el del futuro cercano no es solo alguien que tiene acceso a información y que se preocupa de estar informado. Es también una persona "globalizada" que se desplaza geográficamente, que busca gestionar su enfermedad y su tratamiento, 
que pretende soluciones rápidas, y que no reconoce sus grandes dificultades para interpretar críticamente la información a la que accede. El prototipo es el de una persona exitosa, emprendedora, empoderada y que como enfermo se le reconoce como un "e-Patient". Entre sus hábitos más recientes está la búsqueda de soluciones como el "plush care" o "doctors on demand" que es un sistema de consulta por telefonía celular, a un médico desconocido tipo "call-center", quien puede solicitar exámenes enviando muestras biológicas, con acceso directo a sus resultados y quien finalmente prescribe conductas e indicaciones para tratar su dolencia ${ }^{5}$. Los llamados pacientes siglo 21 son también personas que usan cada vez más dispositivos electrónicos llamados "wearables" que miden su ritmo y frecuencia cardíaca, consumo calórico, saturación de oxígeno o presión arterial, datos que se pueden transmitir directamente a su computadora, a su ficha o registro clínico, o a bases de datos para proyectos aun indeterminados. Hay también cajas organizadoras de medicamentos que envían señales para informar al centro de salud si el enfermo ingirió o no cada fármaco indicado. Otra innovación es el desarrollo de múltiples aplicaciones para "Smartphone" llamadas "Health APP", que ayudan a controlar ejercicios, nutrición y estilos de vida y que permiten el control de tratamientos enviando datos a registros clínicos o a bases de datos para investigación. La relevancia, utilidad y el respeto a la confidencialidad de la información recogida en estos sistemas son temas en discusión, razón por la cual muy pocas de estas aplicaciones han recibido aprobación de la FDA: U.S. Food and Drug Administration ${ }^{6}$. Sin embargo, ya están disponibles en el mercado.

El paciente siglo 21 se enfrenta también a nuevos escenarios y posibilidades de diagnósticos y tratamientos relacionados con la medicina predictiva o personalizada, a estudios y posibles intervenciones genéticas con interpretaciones y consecuencias de largo plazo no bien conocidas, a diversas intervenciones o medicación para el mejoramiento de las personas o de sus hijos que están por nacer, a nuevas y variadas alternativas de reproducción humana, y finalmente a la posibilidad de gestionar no solo su vida sino también su muerte accediendo a diversas formas de muerte médicamente asistida. Muchas de estas posibilidades están aun en investigación o no han pasado la prueba del tiempo para asegurar sus beneficios y la ausencia de riesgos. Sin embargo, son alternativas atractivas, que la medicina actual ya ofrece y que las personas solicitan, valorándolas de manera positiva y esperanzadora sin haberlas analizado con el necesario juicio crítico.

Cabe señalar que mientras aumenta el número de pacientes más informados y conectados a esta creciente gama de avances tecnológicos, la expectativa de vida de la población aumenta mundialmente aunque no así la expectativa de vida saludable, con el consecuente aumento exponencial del número de personas mayores con enfermedades crónicas y limitaciones de muy difícil control. Actualmente estas personas en general no son las que acceden a los avances tecnológicos descritos, lo cual constituye un serio problema de justicia social.

La perspectiva expuesta no debería ser percibida como negativa o amenazante, sino más bien como un positivo cambio histórico. Los progresos de la medicina constituyen un gran beneficio para las personas en su salud, con más y mejores oportunidades de tratamiento de enfermedades y de condiciones incapacitantes, de prevención de enfermedades, de mejores estilos de vida y de mayor expectativa de vida. Los enfermos actuales y futuros, con mayor y mejor información deberían por lo tanto llegar a ser personas más responsables de su autocuidado, con mejores estilos de vida, con mayor prevención de enfermedades o de sus complicaciones, y con mejor observancia de las indicaciones y de los tratamientos demostradamente eficaces. En síntesis el paciente del siglo 21, aunque tenga una relación clínica diferente, con una confianza más limitada en la medicina y en los médicos, es o será un mejor paciente, más informado y más responsable de su salud ${ }^{7}$. Sin embargo, cada paciente, cuando está enfermo, mantiene las mismas necesidades existenciales y de cuidado que tuvieron los pacientes de todas las épocas, y los profesionales de la salud tenemos el mismo deber moral de relacionarnos bien con cada uno de ellos y de ayudarlos en nuestros respectivos ámbitos de acción.

De lo expuesto se puede concluir o proponer que la relación clínica actual, y la del futuro predecible, ha de ser una relación entre el médico, o entre grupos de profesionales médicos y no médicos, con pacientes no tradicionales y con sus grupos familiares. Tienen y tendrán las características que hemos descrito y con ellos se necesitará tomar decisiones compartidas que sean prudentes y razonables frente a alternativas muy diversas y cambiantes, muchas de ellas no claramente demostradas. Lo 
anterior requiere formación en valores y en fundamentos éticos, no solo de los médicos y de los otros profesionales de la salud, sino también de los ciudadanos y de los pacientes del siglo 21, a través de programas de educación social en Bioética. Esta es una gran responsabilidad, aun muy escasamente asumida, de las instituciones académicas y de las instituciones de salud, a través de los Centros de Bioética y de los Comités de Ética Asistencial.

Dr. Juan Pablo Beca Infante Profesor Titular de Universidad del Desarrollo, Director del Magíster Interuniversitario en Bioética Facultad de Medicina Clínica Alemana-Universidad del Desarrollo, Miembro Honorario de la Academia Chilena de Medicina Email:jpbeca@udd.cl

\section{Bibliografía}

1.- EMANUEL EJ, EMANUEL LL. Four models of the physician-patient relationship. JAMA 1992; 267: 22216.

2.- LAZARO J, GRACIA D. La relación médico-enfermo a través de la historia. An Sist Sanit 2006; 29 (supl 3): 7-17. Disponible en: http://scielo.isciii.es/scielo. php?script $=$ sci_pdf\&pid=S1137-66272006000600002 \&lng $=$ es\&nrm=iso\&tlng $=$ es

3.- BECA JP. Relación Clínica. Boletín Academia Chilena de Medicina 2015; $n^{\circ}$ 52, pp 385-90. Disponible en https://medicina.udd.cl/centro-bioetica/files/2016/11/
JP-Beca-Relacion-clinica-boletin2015.pdf

4.- Ley 290.584. Ley que regula los derechos y deberes que tienen las personas en relación con acciones vinculadas a su atención en salud. 24 de abril de 2012. Disponible en: https://www.leychile.cl/Consulta/listaresultadosim ple?cadena $=20584$

5.- PLUSH CARE. Disponible en: https://www.plushcare. com/

6.- FDA. Mobile Medical Applications. 2018. Disponible en: https://www.fda.gov/medicaldevices/digitalhealth/ mobilemedicalapplications/default.htm

7.- BECA JP. El paciente del siglo 21. Boletín Academia Chilena de Medicina 2018, ${ }^{\circ} 55$ (En prensa). 